\title{
Improved Formulation for the Optimization of Wind Turbine Placement in a Wind Farm
}

\author{
Zong Woo Geem and Junhee Hong \\ Department of Energy IT, Gachon University, Seongnam 461-701, Republic of Korea \\ Correspondence should be addressed to Zong Woo Geem; geem@gachon.ac.kr
}

Received 19 February 2013; Revised 14 June 2013; Accepted 16 June 2013

Academic Editor: Ming-Hung Hsu

Copyright (C) 2013 Z. W. Geem and J. Hong. This is an open access article distributed under the Creative Commons Attribution License, which permits unrestricted use, distribution, and reproduction in any medium, provided the original work is properly cited.

\begin{abstract}
As an alternative to fossil fuels, wind can be considered because it is a renewable and greenhouse gas-free natural resource. When wind power is generated by wind turbines in a wind farm, the optimal placement of turbines is critical because different layouts produce different efficiencies. The objective of the wind turbine placement problem is to maximize the generated power while minimizing the cost in installing the turbines. This study proposes an efficient optimization formulation for the optimal layout of wind turbine placements under the resources (e.g., number of turbines) or budget limit by introducing corresponding constraints. The proposed formulation gave users more conveniences in considering resources and budget bounds. After performing the optimization, results were compared using two different methods (branch and bound method and genetic algorithm) and two different objective functions.
\end{abstract}

\section{Introduction}

Wind has a power which can be converted into energy (electricity) generated by wind turbines and mechanical power generated by wind mills. Because wind energy is abundant, renewable, and clean without producing greenhouse gas, it can be an alternative to fossil fuels.

In order to cost-efficiently obtain the wind energy, researchers have considered optimization techniques for the layout of wind turbines in wind farms [1-5]. Mosetti et al. [1] first applied genetic algorithm (GA) to this wind turbine position optimization. However, they did not provide detailed numerical information to replicate, while Grady et al. [2] provided full numerical dataset to replicate and compare the results from various algorithms. Emami and Noghreh [3] explained in more detail the physical wake function from continuity equation of mass conservation law. Rasuo and Bengin [4] expanded the application from flat surface into arbitrary configured terrain. Samorani [5] provided a more realistic power function from subdivided wind velocities.

The optimization formulations in the above-mentioned literature have majorly two types of objective functions (cost per unit power and one over power). However, they do not have any budgetary or technical constraint that can efficiently control budget limitation or turbine number. Thus, this study proposes a more realistic optimization formulation for placing wind turbines in a wind farm by considering the available number of turbines and the available budget. Also, the computational results are to be compared with those from the previous literature.

\section{Physical Wake Model}

The previous literature [1-3] has used the following wake decay model for two consecutive turbines (e.g., turbine 0 and turbine 1):

$$
u_{1}=u_{0}\left[1-\frac{2 a}{\left(1+\alpha x / r_{r}\right)^{2}}\right] \text {, }
$$

where $u_{1}$ is wind speed after the turbine $0, u_{0}$ is wind speed before the turbine $0, a$ is axial induction factor, $\alpha$ is entrainment constant, $x$ is distance between two turbines; and $r_{r}$ is rotor radius of turbines ( $=40 \mathrm{~m}$ in this study). 
The axial induction factor $a$ can be obtained using the following relationship:

$$
C_{T}=4 a(1-a),
$$

where $C_{T}$ is turbine thrust coefficient ( $=0.88$ in this study).

The entrainment constant $\alpha$ can be obtained using the following empirical function [1-3]:

$$
\alpha=\frac{0.5}{\ln \left(z / z_{0}\right)},
$$

where $z$ is hub height of the turbine ( $=60 \mathrm{~m}$ in this study) and $z_{0}$ is surface roughness $(=0.3 \mathrm{~m}$ in this study).

More fundamentally, the wake decay model in (1) can be derived from a continuity equation for mass conservation [3]. If the wind speed immediately after the turbine is assumed to be $1 / 3$ of the original speed, the following continuity equation can be formulated:

$$
\rho \frac{u_{0}}{3} A_{r}+\rho u_{0}\left(A_{1}-A_{r}\right)=\rho u A_{1} .
$$

Here, $\rho$ is wind density which is represented as mass divided by volume. Under the mass conservation law, the total mass directly passing a wind turbine rotor $\left(\rho\left(u_{0} / 3\right) A_{r}\right)$ and nearly passing the rotor $\left(\rho u_{0}\left(A_{1}-A_{r}\right)\right)$ should be equal to the total mass $\left(\rho u A_{1}\right)$ at the distance of $x$. Because $A_{r}=\pi r_{r}^{2}$, $A_{1}=\pi r_{1}^{2}$, and $r_{1}=\alpha x+r_{r}$, (4) can be substituted with (1). Here, $r_{1}$ is downstream rotor radius and $a$ (axial induction factor) is assumed to be $1 / 3$. For (1), this study uses $r_{1}$ instead of $r_{r}$ in order to fairly compare this study with previous ones [1-3] although it is not exact.

\section{Cost and Power Functions}

The total cost of all turbines in a wind farm can be calculated with the following function [1-3]:

$$
C_{t}=N\left(\frac{2}{3}+\frac{1}{3} e^{-0.00174 N^{2}}\right)
$$

where $N$ is the total number of turbines in a wind farm.

The total power (kW-year) generated from all turbines can be calculated with the following function $[2,3]$ :

$$
P_{t}=\sum_{i}^{N} 0.3 u_{i}^{3},
$$

where $u_{i}$ is the wind speed at each turbine.

\section{New Optimization Formulation}

For the objective function of this optimal wind turbine placement problem, researchers have proposed two different types:

$$
\begin{gathered}
\text { Minimize } z_{1}=w_{1} \frac{C_{t}}{P_{t}}+w_{2} \frac{1}{P_{t}} \\
\text { Minimize } z_{2}=\frac{C_{t}}{P_{t}} .
\end{gathered}
$$

Equation (7), proposed by Mosetti et al. [1] and Emami and Noghreh [3], combines two objective functions (cost per unit power and one over power). The objective function of cost per unit power is designed to find the most cost-effective (or minimal cost per unit power) solution while the objective function of one over power is designed to find the most power-effective (or maximal power) solution. Meanwhile, (8), proposed by Grady et al. [2], uses only one objective function.

Although Emami and Noghreh claimed that (7) is better than (8), the former has the following limitations: (1) because the weights $\left(w_{1}, w_{2}\right)$ are arbitrarily assigned by users, the proper setting of the weight values requires tedious trial-anderror process for users; (2) nonetheless, it cannot specifically control budget limit or turbine number; (3) more importantly, it does not significantly improve the performance of (8) (this point will be discussed later). In this sense, (8) is actually better than (7) because the former is simple and easy to use and does not require tedious extra process.

Here, for both equations, the following modifications can be considered to avoid any divided-by-zero error, which happens if searching methods start with $P_{t}=0$ :

$$
\begin{gathered}
\text { Minimize } z_{1}=w_{1} \frac{C_{t}}{P_{t}+\Delta}+w_{2} \frac{1}{P_{t}+\Delta}, \\
\text { Minimize } z_{2}=\frac{C_{t}}{P_{t}+\Delta},
\end{gathered}
$$

where $\Delta$ is tiny amount (e.g., $\Delta=0.0001$ ).

In order to consider exact turbine number or budget limit, the following additional constraints can be also introduced:

$$
\begin{aligned}
& N=N_{a} \\
& C_{t} \leq C_{a} .
\end{aligned}
$$

Equation (11) denotes that the total number of turbines in a wind farm should be equal to the available number of turbines, $N_{a}$. Equation (12) denotes that the total cost of turbines installed in a wind farm should be less than or equal to the available budget, $C_{a}$.

\section{Numerical Example}

The above-mentioned optimization formulation was applied to a popular wind turbine placement problem [1-3] as shown in Figure 1. The wind farm, where wind flows from the top into the bottom with a uniform speed of 12 meter per second, has 100 candidate turbine locations and each location has an area of $200 \mathrm{~m} \times 200 \mathrm{~m}$. For the optimization, each location has a binary value. If the binary variable has a value of 1 , it means a turbine is installed at the location. Otherwise, it means a turbine is not installed. Actually, this is a huge combinatorial problem because total searching space is $2^{100} \approx 1.3 \times 10^{30}$.

For this large-scale problem, a metaheuristic approach, GA, has been actively applied [1-3]. This is because traditional branch and bound (B\&B) method does not give good results. When this study applied $B \& B$ method (commercial code named Solver) to the problem, the objective function 


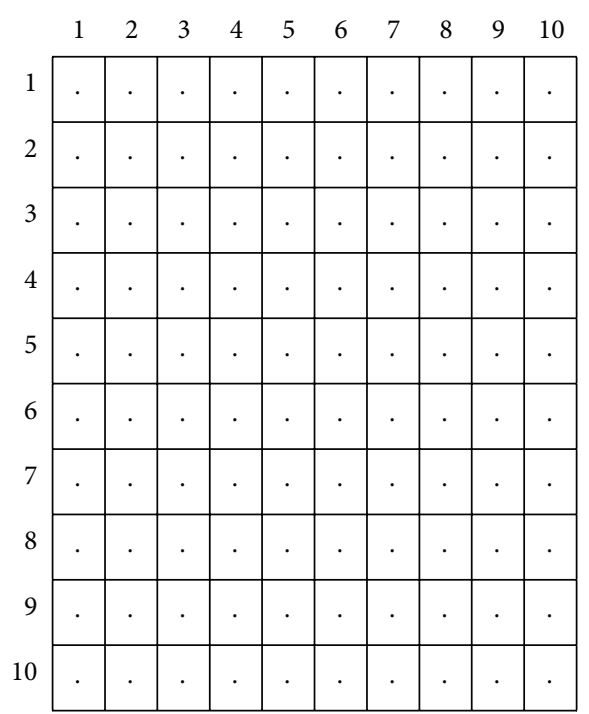

Figure 1: Schematic of wind farm.

TABLE 1: Comparison of results from two objective functions.

\begin{tabular}{lcccc}
\hline $\begin{array}{l}\text { Function } \\
\text { type }\end{array}$ & $\begin{array}{c}\text { No. of } \\
\text { function } \\
\text { evaluations }\end{array}$ & $\begin{array}{c}\text { Time taken } \\
\text { (mm:ss) }\end{array}$ & $w_{1}$ & $w_{2}$ \\
\hline Equation (10) & 130,883 & $18: 07$ & NA & NA \\
& 136,360 & $19: 07$ & 0.5 & 0.5 \\
Equation (9) & 126,765 & $15: 01$ & 0.3 & 0.7 \\
& 347,547 & $40: 41$ & 0.7 & 0.3 \\
\hline
\end{tabular}

value of 2.2824 (unit: unit cost $\times 10^{-3}$ ) was obtained with the cost of 34.18 and the power of 14977.44 (kW/year) as shown in Figure 2(a) $(N=51)$. Meanwhile, GA approach (commercial code named Evolver) found the objective function value of 1.5579 with the cost of 22.09 and the power of 14178.82 as shown in Figure 2(b) $(N=30)$. The result of GA is much better than that of $B \& B$ because the former's generation efficiency is 641.8 (unit: $\mathrm{kW} /$ year/unit cost) while the latter's generation efficiency is 438.2 .

The above GA approach utilized (10) as an objective function. However, (9) was also tested with different weight values $\left(w_{1}=0.5, w_{2}=0.5 ; w_{1}=0.3, w_{2}=0.7\right.$; and $w_{1}=0.7, w_{2}=0.3$ ) as shown in Table 1. When (10) was compared with (9), the former outperformed the latter in two cases $\left(w_{1}=0.5, w_{2}=0.5\right.$ and $\left.w_{1}=0.7, w_{2}=0.3\right)$ in terms of number of function evaluations while all four approaches found the identical global optimum $\left(C_{t}=22.09\right)$ as shown in Figure 2(b). It should be noted that because $C_{t} / P_{t}$ in (9) (or in (7)) is greater than $1 / P_{t}$ by $C_{t}$ times, the approach with bigger $w_{2}$ performed better which considers more balance between $C_{t} / P_{t}$ and $1 / P_{t}$.

As stated previously, in order to more efficiently consider turbine number or budget limit, corresponding constraints should be introduced to the optimization formulation. Table 2 shows the results of GA approaches with different available numbers of turbines, also shown in Figure 2(b) $(N=30)$, Figure 3(a) $(N=10)$, Figure 3(b) $(N=20)$,

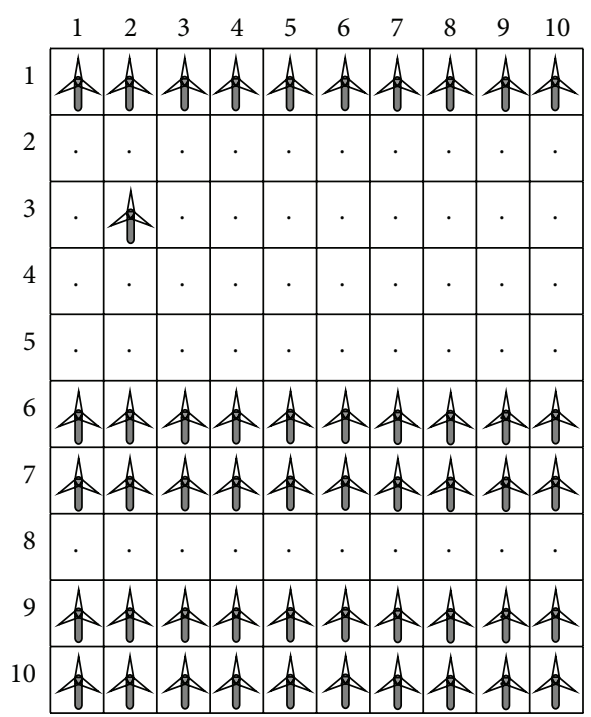

(a)

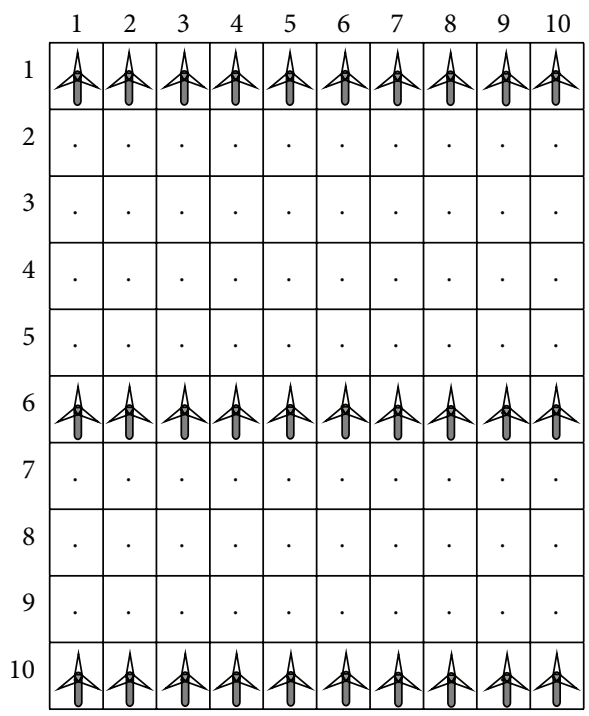

(b)

FIGURE 2: Best results from branch and bound method and genetic algorithm.

TABLE 2: Comparison of results from different turbine numbers.

\begin{tabular}{cccc}
\hline$N$ & $C_{t} / P_{t}\left(10^{-3}\right)$ & $C_{t}$ & $P_{t}$ \\
\hline 10 & 1.8286 & 9.47 & $5,177.64$ \\
20 & 1.6332 & 16.66 & $10,198.82$ \\
30 & 1.5579 & 22.09 & $14,178.82$ \\
40 & 1.6745 & 27.49 & $16,417.49$ \\
\hline
\end{tabular}

and Figure 3(c) $(N=40)$. The objective function becomes minimal (1.5579) when $N$ is 30 , while the second best solution was obtained with $N=20$; the third best solution was obtained with $N=40$; and the fourth best solution was obtained with $N=10$.

By considering the budget limit in (12), the optimal function value of 1.5978 was obtained with the cost of 19.48 


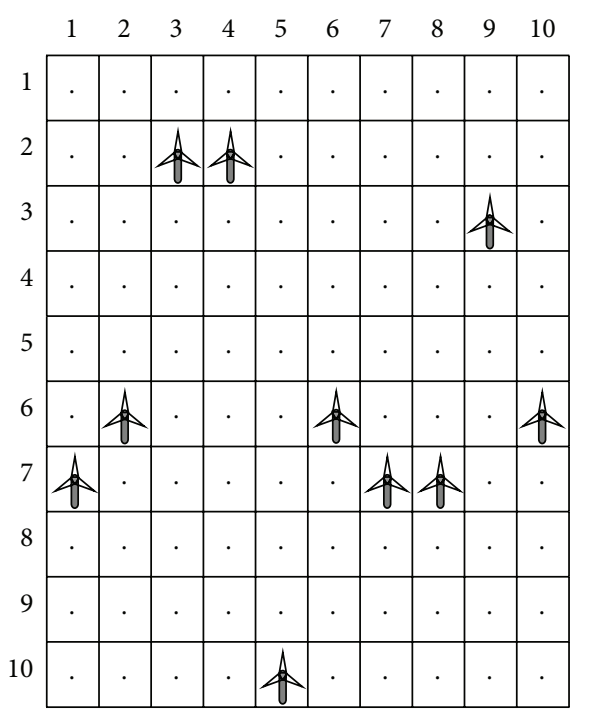

(a)

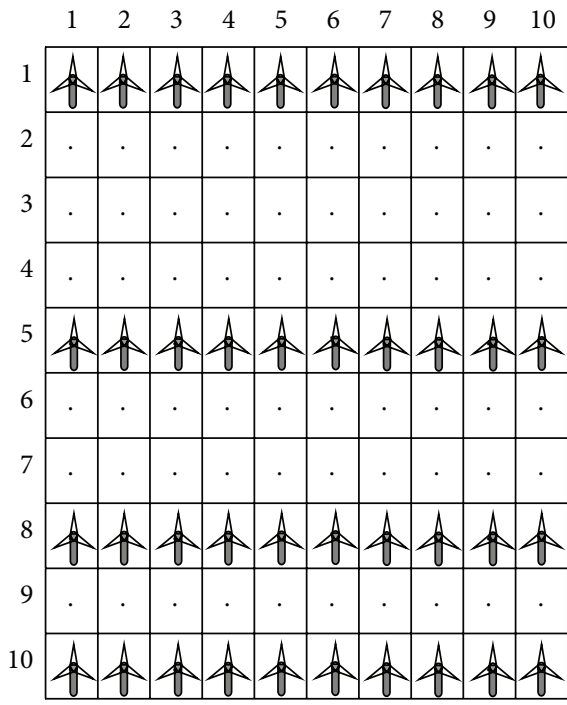

(c)

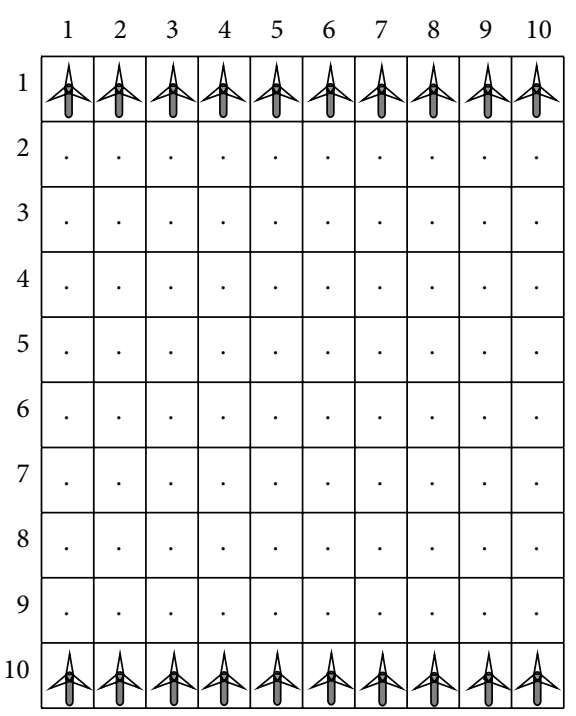

(b)

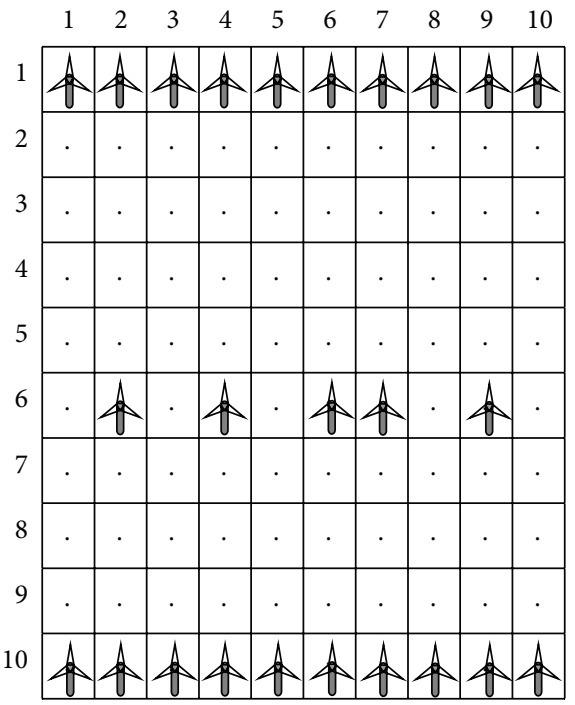

(d)

FIGURE 3: Results of genetic algorithm with different constraints.

and the power of 12,188.82 if available budget $C_{a}$ is 20 . Figure 3(d) shows the corresponding result $(N=25)$.

Previous researches [1-3] did not consider the above turbine number and budget limit constraints. However, these constraints can be efficiently utilized under the limited resources or expenditures.

When the best solution in this study $\left(C_{t} / P_{t}=1.5579 \times\right.$ $10^{-3}$ when $N=30$ ) was compared with other solutions, it outperformed the result by Mosetti et al. [1] according to the comparison by Grady et al. [2]. Also, the best solution here is identical to those by Grady et al. [2] and Emami and Noghreh [3]. However, in terms of the number of objective function evaluations, this study outperformed that by Grady et al. [2] because this study found the best solution after 126,765 evaluations while that by Grady et al. found the identical one after 721,800 evaluations (600 individuals and
1203 generations). Although Emami and Noghreh [3] found the identical best solution, a fair comparison cannot be made because they did not provide the number of total evaluations.

\section{Conclusions}

This study improved the existing optimization formulation for wind turbine placement in a wind farm by introducing (1) nonzero denominator, (2) budget constraint, and (3) resource constraint (number of available turbines). Also, two existing objective functions were compared. In addition, the reason why GA, rather than traditional $B \& B$ method, has vigorously tackled the problem was demonstrated. That is because GA obtained much better result than $B \& B$ for this huge enumeration problem $\left(1.3 \times 10^{30}\right)$. 
When the results were compared, the best solution in this study outperformed those from previous studies (Mosetti et al. [1] and Grady et al. [2]). Although all approaches, including this study, utilized GA techniques, the variant here appeared better than other variants.

Hopefully, future research will include more complicated and practical issues such as minimum power requirement, territory topography, wind direction, wind velocity variability, and power generation by factory-made turbine. Also, other metaheuristic algorithms such as ant colony optimization (ACO) [6] or harmony search (HS) [7] are expected to tackle the problem.

\section{Acknowledgment}

This work was supported by the Gachon University research fund of 2013 (GCU-2013-R166).

\section{References}

[1] G. Mosetti, C. Poloni, and B. Diviacco, "Optimization of wind turbine positioning in large windfarms by means of a genetic algorithm," Journal of Wind Engineering and Industrial Aerodynamics, vol. 51, no. 1, pp. 105-116, 1994.

[2] S. A. Grady, M. Y. Hussaini, and M. M. Abdullah, "Placement of wind turbines using genetic algorithms," Renewable Energy, vol. 30, no. 2, pp. 259-270, 2005.

[3] A. Emami and P. Noghreh, "New approach on optimization in placement of wind turbines within wind farm by genetic algorithms," Renewable Energy, vol. 35, no. 7, pp. 1559-1564, 2010.

[4] B. Rasuo and A. C. Bengin, "Optimization of wind farm layout," FME Transactions, vol. 38, pp. 107-114, 2010.

[5] M. Samorani, "The wind farm layout optimization problem," Tech. Rep., Leeds School of Business, University of Colorado at Boulder, Boulder, Colo, USA, 2010.

[6] M. Dorigo, V. Maniezzo, and A. Colorni, "Ant system: optimization by a colony of cooperating agents," IEEE Transactions on Systems, Man, and Cybernetics, Part B, vol. 26, no. 1, pp. 29-41, 1996.

[7] Z. W. Geem, J. H. Kim, and G. V. Loganathan, "A new heuristic optimization algorithm: harmony search," Simulation, vol. 76, no. 2, pp. 60-68, 2001. 


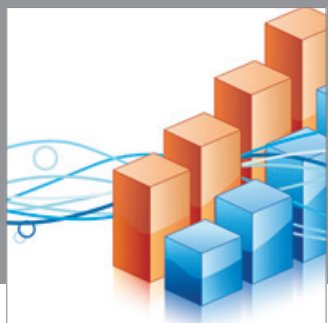

Advances in

Operations Research

mansans

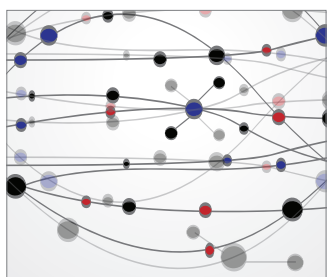

The Scientific World Journal
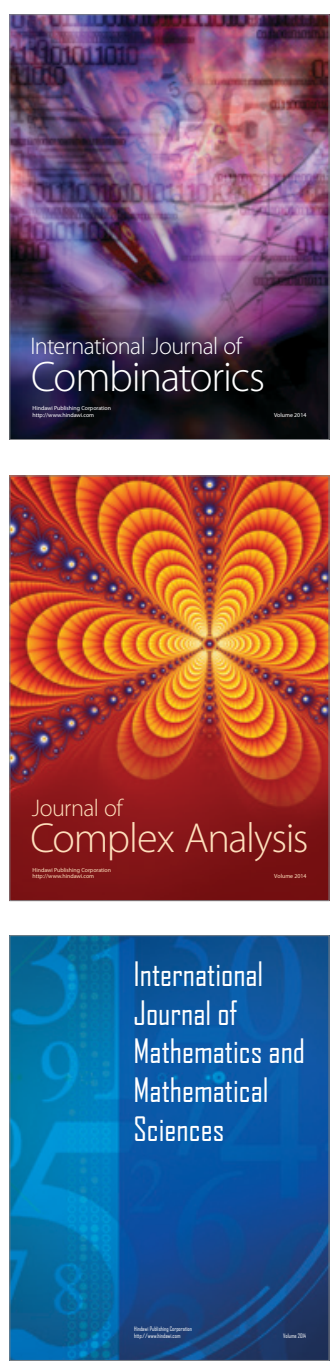
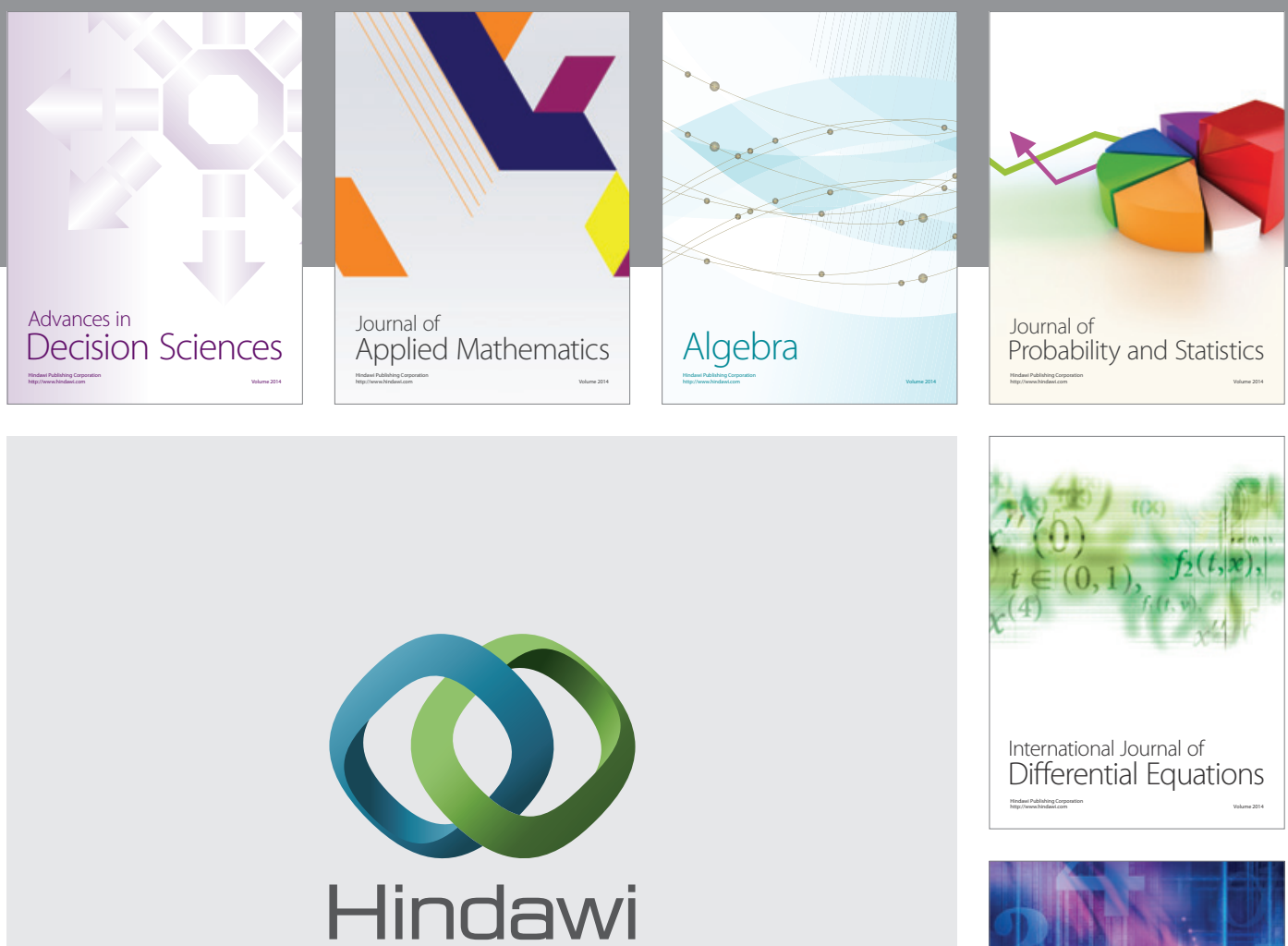

Submit your manuscripts at http://www.hindawi.com
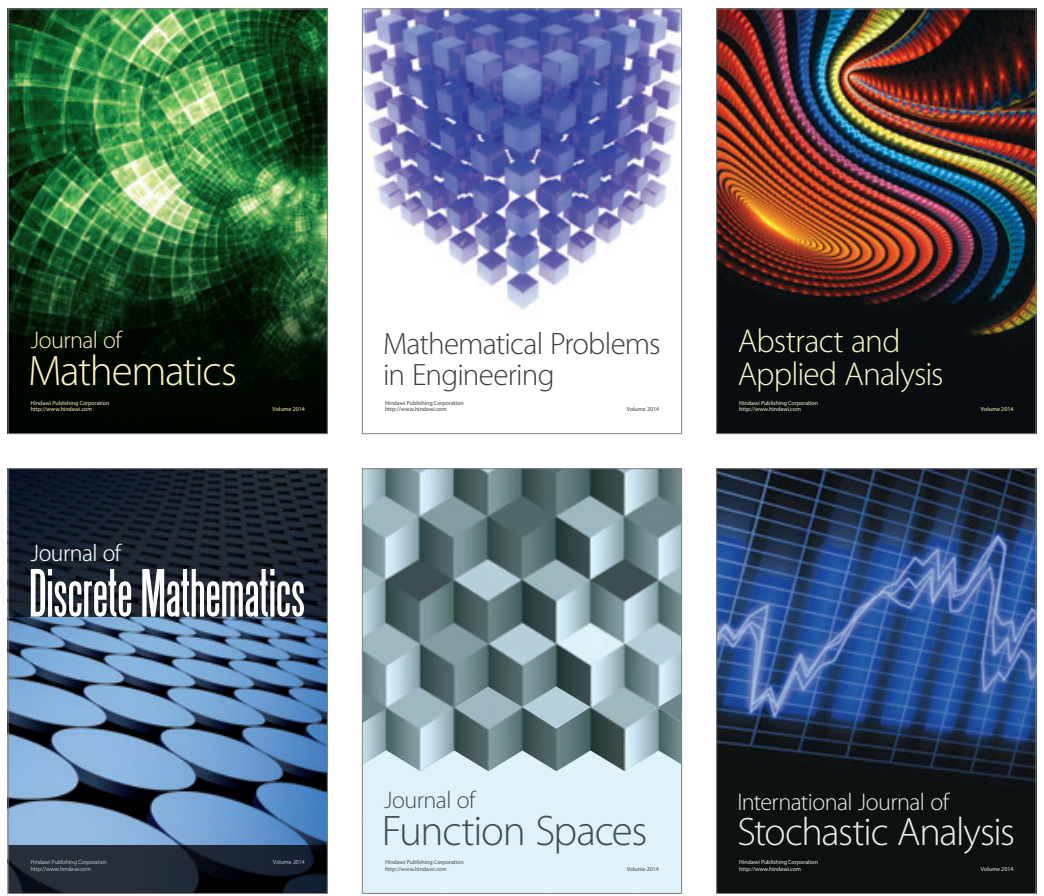

Journal of

Function Spaces

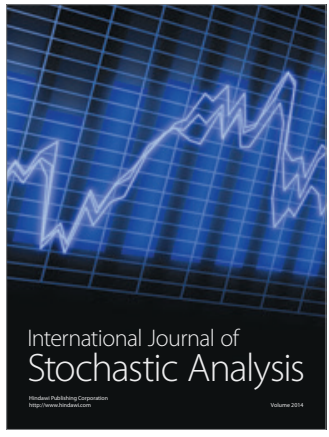

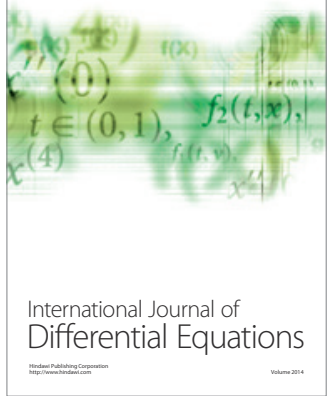
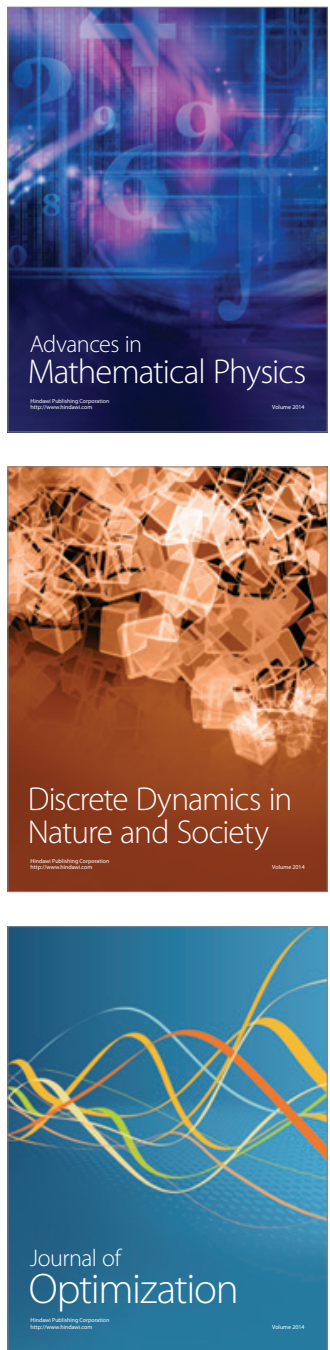